\title{
Correction to: Long-term solar activity and terrestrial connections. Part II: at the beckon of the sun?
}

\author{
N. D. Diamantides \\ Department of Geography, Kent State University, P.O. Box 5190, Kent, Ohio 44242, USA
}

Received: 25 March 1996 / Revised: 16 August 1997 / Accepted: 5 September 1997

Published in Annales Geophysicae, Volume 16, number 5, on pages 492-509, 1998

The author would like to apologize for the omission of a figure in the above-mentioned paper. The missing figure is shown in the right column.

The following sentence from page 499, Part II, "In the meantime, the autocorrelogram of the residual of the preceding regression, seen in Fig. 11 reveals....." should now read,

"In the meantime, as shown in Fig. 10a, the autocorrelogram of the residual reveals the presence of the 8-year periodicity found in $D z_{11}(t)$, Eq. (52) Part I."

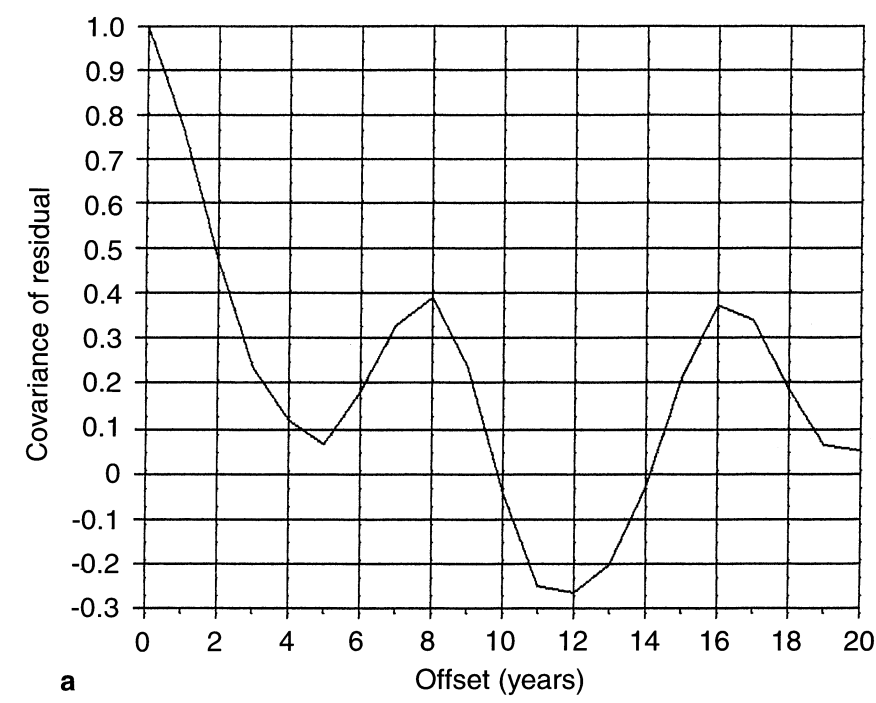

Fig. 10a. Covariance of residual 